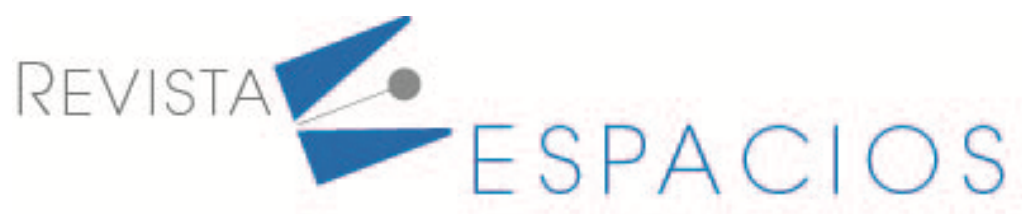

\title{
Criterios de usabilidad para la evaluación de repositorios institucionales de investigación
}

\author{
Usability criteria for the evaluation of institutional research repositories
}

\author{
ALVITES, Pedro A. ${ }^{1}$ \\ CHANCHÍ, Gabriel E. ${ }^{2}$ \\ DE LA CRUZ, Percy E. ${ }^{3}$
}

\begin{abstract}
Resumen
Uno de los medios más importantes para almacenar, usar y divulgar diferentes recursos académicos son los repositorios de investigación. Con el propósito de mejorar el diseño centrado en el usuario en estos repositorios, se propuso como aporte un conjunto de criterios de verificación categorizados en siete heurísticas de usabilidad. Mediante estos criterios y heurísticas, se desarrolló una herramienta de evaluación que fue utilizada para la conducción de una inspección sobre el repositorio académico de la Universidad de Lima, permitiendo obtener un conjunto de recomendaciones para mejorar la usabilidad del repositorio.
\end{abstract}

Palabras clave: Criterios de usabilidad, heurísticas, repositorios institucionales, usabilidad.

\begin{abstract}
One of the most important means of storing, using and disseminating different academic resources are research repositories. In order to improve user-centered design in these repositories, we proposed as a contribution a set of verification criteria categorized in seven usability heuristics. Using these criteria and heuristics, we developed an evaluation tool that was used to conduct an inspection of the academic repository of the Universidad de Lima, allowing us to obtain a set of recommendations to improve the usability of the repository.
\end{abstract}

key words: Heuristics, institutional repositories, usability, usability criteria.

\section{Introducción}

El área de la interacción humano computador, se enfoca en el diseño, desarrollo, implementación y evaluación de sistemas informáticos acordes a las necesidades de un usuario (Chao, 2009). Esta área cobra gran importancia en la actualidad, gracias a la gran cantidad de usuarios que consumen diversos tipos de aplicaciones en diferentes contextos de aplicación. Así, la vinculación de la usabilidad en las diferentes fases del proceso de desarrollo se ha convertido en un elemento prioritario en el área de la ingeniería del software (Grau, 2007; Sanchez, 2011; Solis Tech, 2016).

Los sistemas usables hacen referencia a aquellos que son fáciles de usar y de aprender, por lo que el concepto de usabilidad no es propio del área de los sistemas informáticos y está relacionado directamente con la psicología

\footnotetext{
${ }^{1}$ Profesor del Centro de Informatica y Sistemas. Universidad Cesar Vallejo - Sede Los Olivos - Lima. palvitesh@ucvvirtual.edu.pe

2 Profesor del Programa de Ingeniería de Sistemas. Facultad de Ingeniería. Universidad de Cartagena. gchanchig@unicartagena.edu.co

3 Profesor principal - Facultad de Ingeniería de Sistemas e Informática. Universidad Nacional Mayor de San Marcos. pdelacruzv@unmsm.edu.pe
} 
cognitiva (Preece, 1994). Según Nielsen (2012) la usabilidad es entendida como un atributo de calidad que mide la facilidad de la interacción entre el usuario y la interfaz. Del mismo modo, de acuerdo al estándar ISO 9241-11, la usabilidad es el grado en el que un producto puede ser utilizado por usuarios específicos para conseguir objetivos específicos con efectividad, eficiencia y satisfacción en un determinado contexto de uso (Chanchí, 2019; Enriquez \& Casas, 2013; Martins, 2013; Weichbroth, 2020).

Un repositorio institucional de investigación es aquel conjunto de servicios prestados por las universidades o centros de investigación a su comunidad para recopilar, gestionar, difundir y preservar su producción científica digital a través de una colección organizada, de acceso abierto e interoperable (Azorín Millaruel et al., 2017). Los repositorios institucionales reúnen la producción intelectual de los miembros de una institución académica resultado de su actividad docente e investigadora, desarrollado por la propia institución para ofrecer un conjunto de servicios a su comunidad, incluyendo el almacenamiento, gestión, acceso abierto, distribución y preservación de los recursos depositados en ella. Abarcan un amplio rango de información digital, desde artículos científicos a tesis doctorales, publicaciones periódicas de producción propia, materiales educativos, datasets (datos en bruto resultado de una investigación), objetos multimedia, etc.(Bailey, 2005; Hernández Pérez, 2007).

Del mismo modo, los repositorios institucionales pueden ser entendidos como estructuras web interoperables de servicios informáticos, dedicadas a difundir la perpetuidad de los recursos científicos y académicos (físicos o digitales) de las universidades, a partir de la enumeración de un conjunto de datos específicos (metadatos), para que esos recursos se puedan recopilar, catalogar, acceder, gestionar, difundir y preservar de forma libre y gratuita, de manera que están estrechamente ligados a los ideales y objetivos del acceso abierto (Duperet Cabrera, 2015).

Dado que los repositorios institucionales están pensados para ser dirigidos a un público diverso y masivo, es fundamental que dichos repositorios estén dotados de características de usabilidad. De este modo, en este estudio se propone un conjunto de heurísticas y criterios de usabilidad para el diseño, desarrollo y evaluación de repositorios institucionales. Las heurísticas y los criterios de verificación propuestos permiten complementar y especificar las heurísticas presentadas en Azorín et. al. (2017), de tal modo que posibilitan la obtención de resultados más precisos en la conducción de inspecciones de usabilidad, dado que los criterios asociados a cada una de las heurísticas permiten evaluar de manera más exacta el alcance de cada una de las heurísticas. Así mismo, los criterios de evaluación definidos son un aporte en la evaluación de sitios web destinadas a albergar respositorios académicos, dado que los métodos convencionales de evaluación de usabilidad de sitios web no consideran en sus heurísticas y criterios las especificidades de los repositorios académicos institucionales (Hurtado et al., 2018).

Así mismo, como contribución adicional, se propone una herramienta para la conducción de inspecciones de usabilidad en el contexto de los repositorios institucionales. La herramienta permite la valoración de los criterios de cada heurística, así como el análisis estadístico y gráfico de los resultados de una determinada prueba.

Una inspección de usabilidad corresponde al método mediante el cual un conjunto de evaluadores explora y califica la usabilidad de un producto software teniendo en cuenta un conjunto de heurísticas y criterios propias del contexto del software analizado. A través de la inspección se buscan obtener problemas de usabilidad, que al ser corregidos contribuyen con la mejora de la experiencia de los usuarios (Enriquez \& Casas, 2013). Así, las heurísticas y la herramienta propuesta permiten servir de apoyo al coordinador de la prueba de cara a la obtención automatizada de resultados de interés en cuanto a la mejora de la calidad del producto.

El resto del artículo está organizado de la siguiente forma: en la sección 2 se presenta las fases metodológicas que guiaron la presente investigación; en la sección 3 se presentan las heurísticas y criterios definidos para el presente estudio; en la sección 4 se describe la herramienta construida para la ejecución de inspecciones de 
usabilidad para repositorios académicos; en la sección 5 se describe un estudio de caso en el cual se aplicaron las heurísticas y criterios propuestos; finalmente en la sección 6 se presentan las conclusiones y trabajos futuros derivados de la presente investigación.

\section{Metodología}

En el desarrollo de la presente investigación, se consideraron 3 fases: definición de heurísticas y criterios de usabilidad para repositorios institucionales, construcción de una herramienta para la ejecución de inspecciones de usabilidad en repositorios institucionales y caso de estudio (ver Figura 1). Esta metodología fue adaptada a partir del trabajo presentado en Chanchí (2020).

Figura 1

Metodología considerada

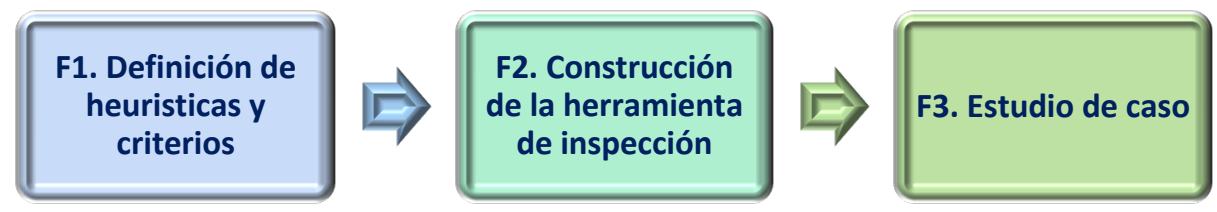

A continuación se describen las diferentes fases de la metodología presentadas en la Figura 1.

Fase 1 - Definición de heurísticas y criterios: En esta fase se extrajeron y se adaptaron las directrices y criterios de la guía para la evaluación de repositorios institucionales de investigación, para lo cual se tuvo en cuenta las heurísticas presentadas en Azorín et. al. (2017).

Fase 2 - Construcción de la herramienta de inspección: A partir de la exploración e identificación realizada en la fase uno, se definieron un conjunto de criterios en términos de preguntas para cada una de las heurísticas extraídas y adaptadas de la guía para la evaluación de repositorios institucionales de investigación (Azorín Millaruel et al., 2017).

Fase 3 - Estudio de caso: Según los criterios definidos en la fase dos, se procedió con la generación de una herramienta de inspección de usabilidad en el lenguaje Java, la cual permite la valoración de los criterios definidos, así como el análisis gráfico sobre el cumplimiento de éstos.

\section{Heurísticas y criterios de usabilidad propuestos}

En esta sección se presentan las heurísticas y criterios definidos para el diseño y evaluación de repositorios institucionales desde la perspectiva de la usabilidad. Estas heurísticas fueron adaptadas a partir de la guía para la evaluación de repositorios institucionales de investigación propuesta en Azorín Millaruel et al. (2017). Cada heurística propuesta tiene asociados un conjunto de criterios que permiten verificar el cumplimiento de dicha heurística, los cuales han sido redactados en términos de pregunta. Cada pregunta puede ser evaluada en una escala de 0 a 5 , siendo 0 el nivel mínimo de cumplimiento y 5 el máximo nivel de cumplimiento. Así mismo, en caso que el criterio no aplique al repositorio evaluado, el evaluador puede escoger la opción N/A (No aplica). A partir de lo anterior, en la tabla 2, se presentan las 7 y heurísticas definidas para el diseño y construcción de repositorios académicos. 
Tabla 1

Heurísticas de usabilidad definidos

\begin{tabular}{|l|l|}
\hline \multicolumn{1}{|c|}{ Heurísticas } & \multicolumn{1}{c|}{ Descripción } \\
\hline H1. Visibilidad & $\begin{array}{l}\text { El repositorio deberá tener presencia en directorios nacionales e internacionales, así } \\
\text { como en la existencia de un nombre normalizado que lo identifique en todos ellos. }\end{array}$ \\
\hline H2. Políticas & El repositorio deberá tener definido sobre la misión y objetivos del repositorio. \\
\hline H3. Aspectos Legales & $\begin{array}{l}\text { Directivas relacionadas con la propiedad intelectual de los contenidos distribuidos en } \\
\text { el repositorio, teniendo en cuenta que dichos contenidos están libres, sin restricciones } \\
\text { en su visualización, descarga y distribución. }\end{array}$ \\
\hline H4. Metadatos & $\begin{array}{l}\text { Definen las características y el formato de los metadatos que deben tener los } \\
\text { documentos del repositorio. }\end{array}$ \\
\hline H5. Interoperabilidad & $\begin{array}{l}\text { Los datos se deben de proveer a través del protocolo OAl-PMH (Open Archive } \\
\text { Initiative-Protocol for Metadata Harvesting), se identifican los recursos de } \\
\text { investigación a través de uno o varios sets, se identifican las publicaciones financiadas } \\
\text { por el estado peruano. }\end{array}$ \\
\hline $\begin{array}{l}\text { H6. Estadísticas } \\
\text { H7. Servicios y } \\
\text { funcionalidades de valor } \\
\text { agregado }\end{array}$ & $\begin{array}{l}\text { El repositorio proporciona un servicio de estadísticas sobre el uso de los recursos } \\
\text { almacenados, contenidos, descargas en un lugar visible. }\end{array}$ \\
$\begin{array}{l}\text { Tener la posibilidad de enlazar con redes sociales, Integración del repositorio con } \\
\text { gestores bibliográficos. El repositorio permite exportar las citas a diferentes } \\
\text { plataformas o gestores bibliográficos (Mendeley, Refworks, Zotero, etc.). }\end{array}$
\end{tabular}

Del mismo modo, en la Tabla 2 se presentan un conjunto de criterios expresados en términos de preguntas y asociados a las 7 heurísticas de usabilidad definidas y mostradas en la Tabla 1.

Tabla 2

Criterios de usabilidad definidos

\begin{tabular}{|c|c|}
\hline Heurísticas & Criterios Definidos \\
\hline H1. Visibilidad & $\begin{array}{l}\text { ¿Existe presencia en directorios nacionales e internacionales? } \\
\text { ¿Existe presencia en recolectores nacionales e internacionales? } \\
\text { ¿Existencia de un nombre normalizado en todos ellos? } \\
\text { ¿Existe iniciativas para fomentar la visibilidad del repositorio dentro de la propia } \\
\text { institución? }\end{array}$ \\
\hline H2. Políticas & $\begin{array}{l}\text { ¿Existe una declaración sobre la misión y objetivos del repositorio? } \\
\text { ¿Existe documento de acceso público, donde especifiqué quién puede depositar? } \\
\text { ¿Existe documento de acceso público, donde se especifique qué se puede depositar y } \\
\text { en qué formatos? } \\
\text { ¿Existe una oferta de contacto y asesoramiento visible? }\end{array}$ \\
\hline H3. Aspectos Legales & $\begin{array}{l}\text { ¿Existe una autorización por el autor o el titular de los derechos que permite la } \\
\text { distribución de contenidos? } \\
\text { ¿Inclusión de la información sobre los derechos de autor en los metadatos puestos a } \\
\text { disposición por el repositorio? }\end{array}$ \\
\hline H4. Metadatos & $\begin{array}{l}\text { ¿Se utiliza el formato de metadatos OAI_DC.? } \\
\text { ¿El repositorio utiliza el campo dc:relation para identificar los proyectos de } \\
\text { investigación financiados? }\end{array}$ \\
\hline H5. Interoperabilidad & $\begin{array}{l}\text { ¿Se proveen los datos a través del protocolo OAI-PMH? } \\
\text { ¿Existe integración con otros sistemas de información de la institución? }\end{array}$ \\
\hline
\end{tabular}




\begin{tabular}{|l|l|}
\hline \multicolumn{1}{|c|}{ Heurísticas } & \multicolumn{1}{c|}{ Criterios Definidos } \\
\hline H6. Estadísticas & $\begin{array}{l}\text { ¿El repositorio proporciona un servicio de estadísticas sobre el uso de los recursos } \\
\text { almacenados? } \\
\dot{E} \text { El repositorio ofrece en un lugar visible estadísticas públicas anuales? }\end{array}$ \\
\hline $\begin{array}{l}\text { H7. Servicios y } \\
\text { funcionalidades de valor } \\
\text { agregado }\end{array}$ & $\begin{array}{l}\text { ¿Existe la posibilidad de enlazar con redes sociales? } \\
\dot{E} \text { Existe integración del repositorio con gestores bibliográficos? }\end{array}$ \\
\hline
\end{tabular}

\section{Herramienta construida}

Con el fin de aplicar de modo más efectivo las heurísticas y criterios propuestos en este trabajo, se desarrolló en el lenguaje de programación Java una herramienta para la conducción de inspecciones de usabilidad para repositorios académicos. Así, en la Figura 2 se presenta la interfaz principal de la herramienta propuesta en este trabajo. La herramienta propuesta obtiene desde un archivo de texto plano las heurísticas y criterios propuestos, de tal modo que por cada heurística definida la herramienta crea una pestaña. De este modo, a modo de ejemplo en la Figura 3, se han cargado desde un archivo las heurísticas y criterios genéricos presentados en la Figura 2.

Figura 2

Herramienta de inspección propuesta

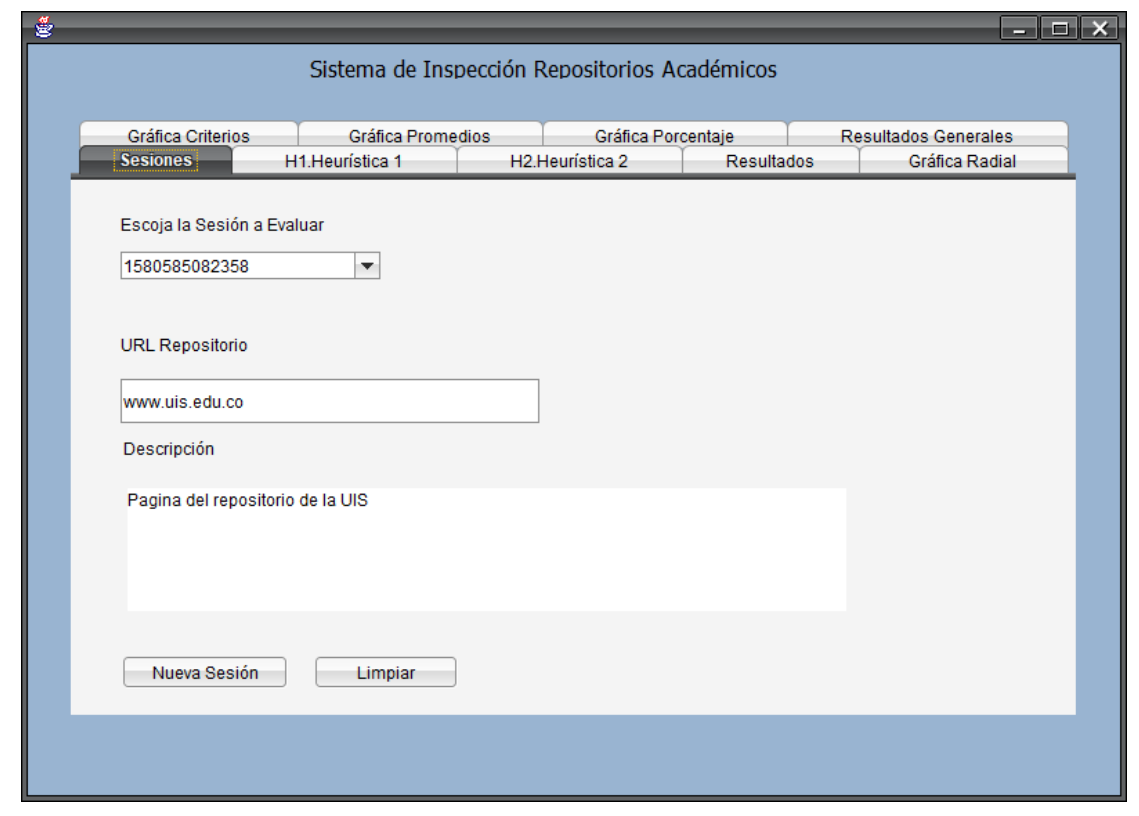

Figura 3

Archivo de configuración

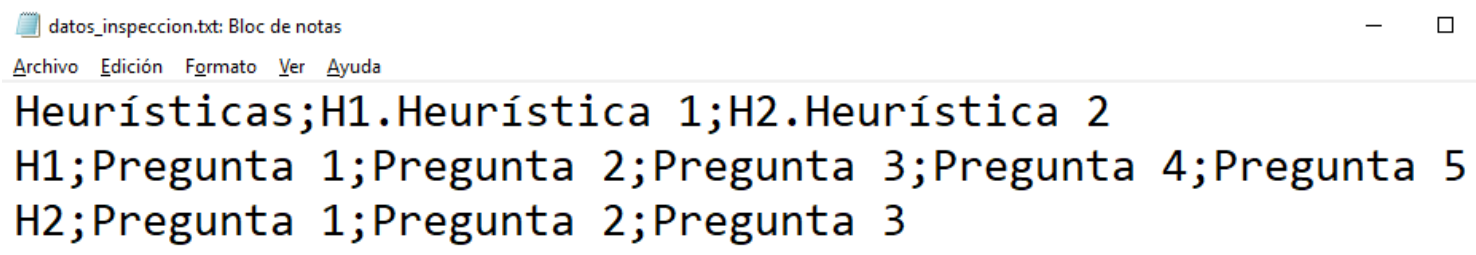

Tal como se observa en la Figura 3 la primera línea contiene los nombres de las heurísticas y desde la segunda línea en adelante son presentados los diferentes criterios asociados a cada heurística en términos de pregunta, 
de tal modo que dichas preguntas permiten evaluar de manera más exacta el cumplimiento de las heurísticas a las que están asociadas. La herramienta permite la creación de sesiones de evaluación (primera pestaña), las cuales son almacenadas en una base de datos portable tipo JSON (Javascript Object Notation) llamada jsondb. Así mismo, tal como se aprecia en la Figura 4, cada pestaña asociada a una heurística permite a los evaluadores la valoración de los criterios pertenecientes a esta (evaluación entre 0 y 5), de tal modo que una vez evaluadas las diferentes heurísticas, es posible obtener los promedios de la evaluación y los promedios generales. Del mismo modo la herramienta permite obtener la gráfica de cada evaluación, así como las gráficas generales de la inspección. De este modo, en la Figura 5 se muestran los diferentes resultados para las dos heurísticas cargadas como ejemplo en esta sección, así como el promedio de las evaluaciones realizadas. El promedio calculado permite determinar el grado de cumplimiento de cada una de las heurísticas definidas a partir de las calificaciones asignadas a cada uno de los criterios de ejemplo.

Figura 4

Pestaña de evaluación de heurísticas

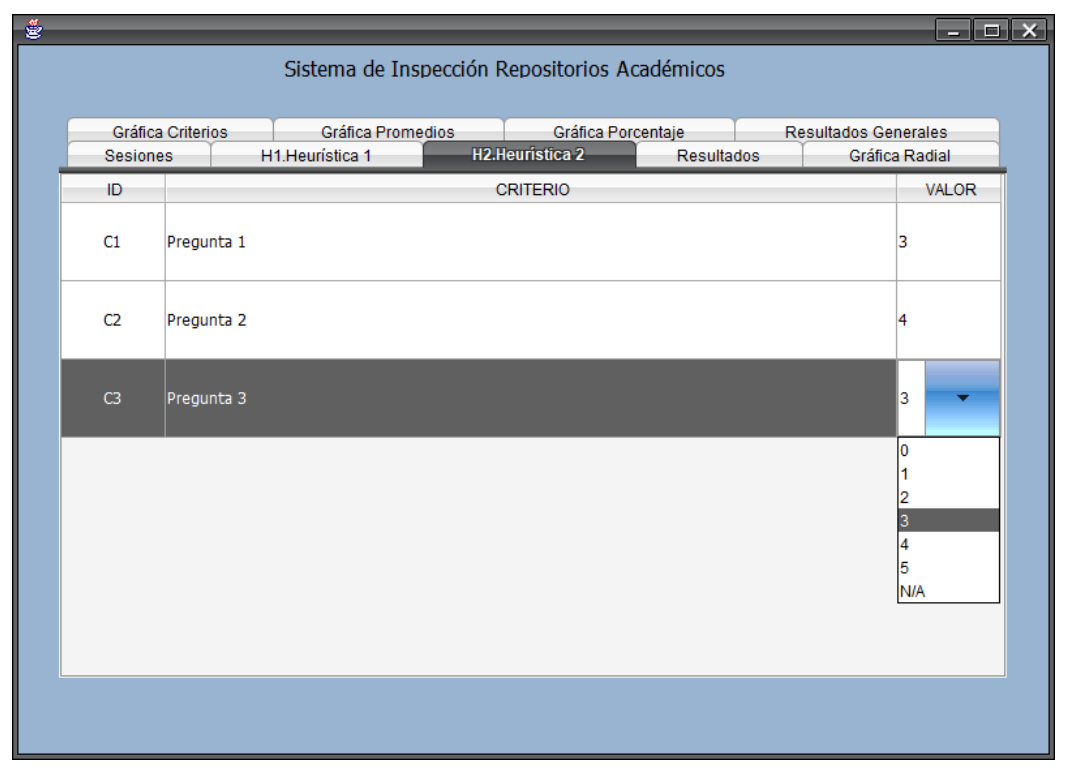

Figura 5

Pestaña de resultados de la inspección

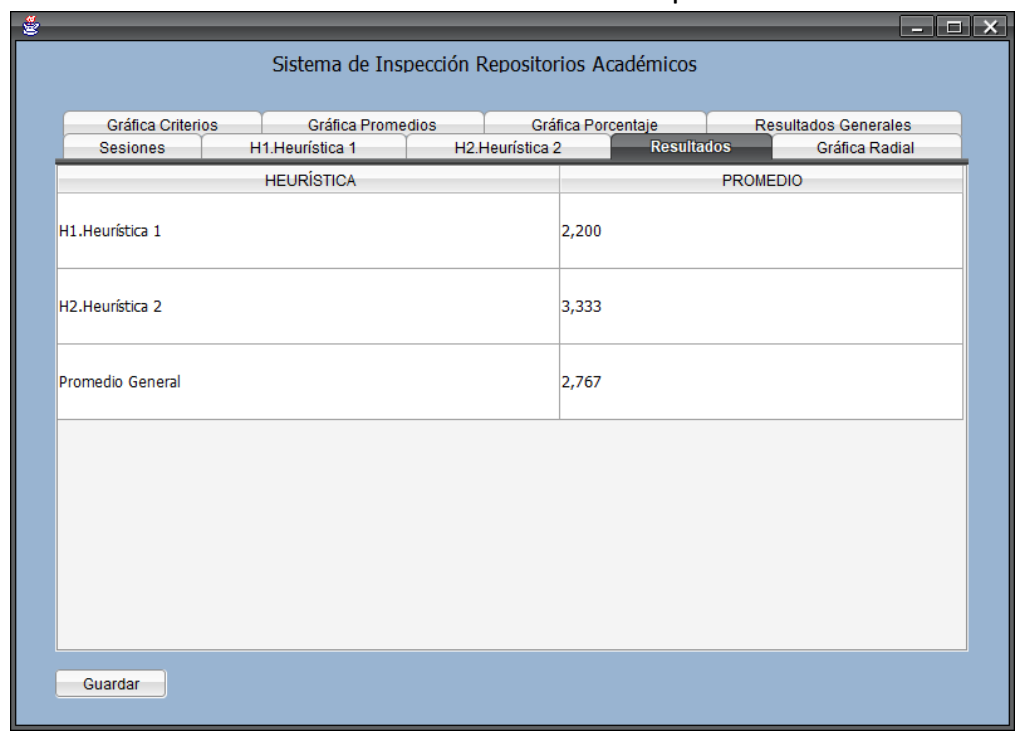


En concordancia con lo anterior, en la Figura 6 se muestran los resultados generales de la evaluación obtenidos para cada criterio de la heurística 1 , los cuales son presentados mediante una gráfica radial. Esta gráfica incluye los datos de las evaluaciones realizadas por un conjunto de evaluadores y también puede ser obtenida para la calificación particular de un solo evaluador.

Figura 6

Nivel de cumplimiento general de la heurística $\mathrm{H} 1$

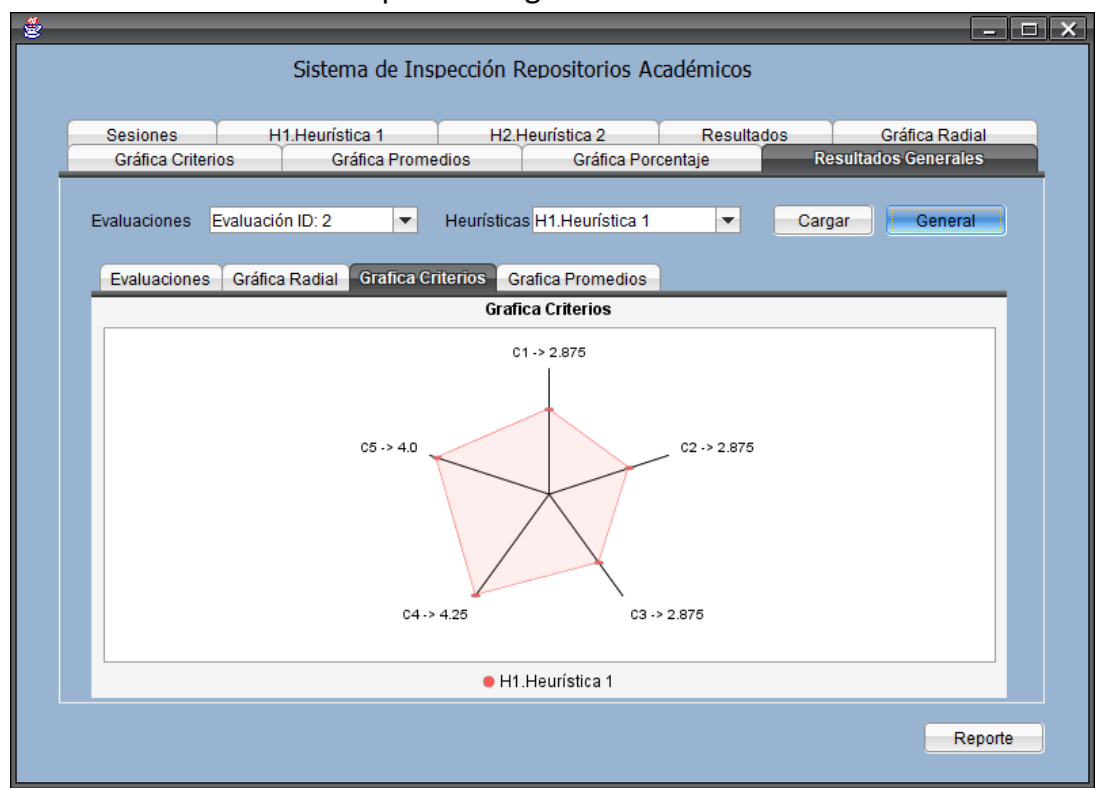

Finalmente, en la Figura 7 se presenta un diagrama de barras arrojado mediante la herramienta, el cual muestra el nivel de cumplimiento de cada una de las heurísticas consideradas en el archivo de configuración.

Figura 7

Nivel de cumplimiento general de las heurísticas

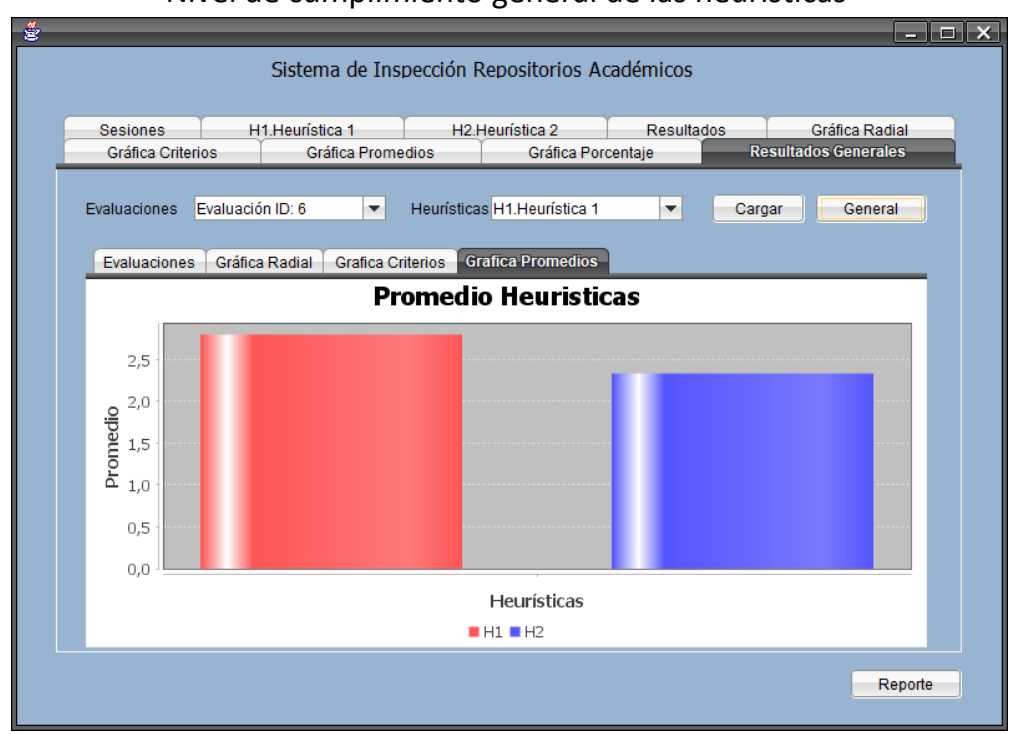




\section{Estudio de caso}

Como medio para verificar la utilidad de las heurísticas y los criterios propuestos, en este artículo se realizó un estudio de caso sobre el repositorio institucional de la Universidad de Lima. En este estudio de caso 3 evaluadores ejecutaron una inspección sobre el repositorio en mención, valorando el cumplimiento de los diferentes criterios y heurísticas mediante el uso de la herramienta propuesta en la sección 4. En la Figura 8 se presenta la tabla de resultados generales generada por la herramienta propuesta.

Figura 8

Repositorio Universidad de Lima

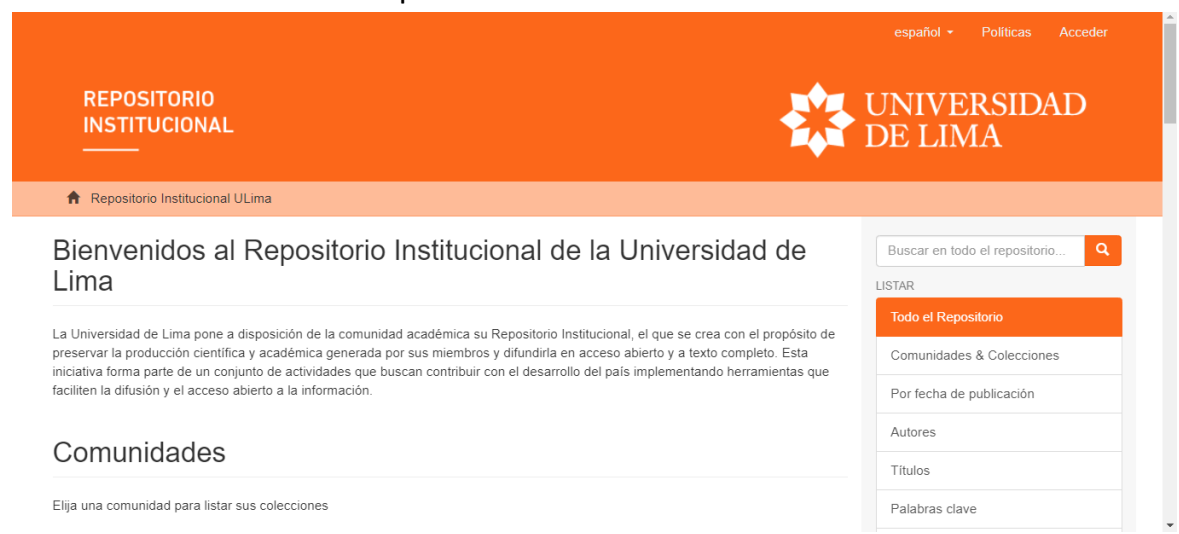

A partir de la inspección de usabilidad realizada al repositorio de la Universidad de Lima, se obtuvieron los resultados presentados en la Tabla 3.

Tabla 3

Heurísticas de usabilidad definidos

\begin{tabular}{|l|c|c|}
\hline \multicolumn{1}{|c|}{ Heurísticas } & Evaluación promedio & $\begin{array}{c}\text { Porcentaje de } \\
\text { cumplimiento }\end{array}$ \\
\hline H1. Visibilidad & 4.875 & 97.5 \\
\hline H2. Políticas & 4.875 & 97.5 \\
\hline H3. Aspectos Legales & 5 & 100 \\
\hline H4. Metadatos & 3 & 60 \\
\hline H5. Interoperabilidad & 2.333 & 46.66 \\
\hline H6. Estadísticas & 3.75 & 75 \\
\hline H7. Servicios y funcionalidades de valor agregado & 4.875 & 97.5 \\
\hline
\end{tabular}

A partir de los resultados de la Tabla 3 es posible observar que de acuerdo a las evaluaciones realizadas a las seis heurísticas definidas, las que menos se cumplen son la de estadísticas (H5) con un porcentaje de cumplimiento del $46.66 \%$ y la de metadata e interoperabilidad $(\mathrm{H} 4)$ con un porcentaje de cumplimiento del $60 \%$. Del mismo modo las heurísticas que mejor se cumplen son las de aspectos legales $(\mathrm{H} 3)$ con un porcentaje de cumplimiento del $100 \%$, la de visibilidad (H1) con un porcentaje de cumplimiento del $97.5 \%$ y la de políticas (H2) con el mismo porcentaje. Lo anterior se puede observar de manera más en la gráfica radial presentada en la Figura 9. 
Figura 9

Nivel general de cumplimiento de las heurísticas

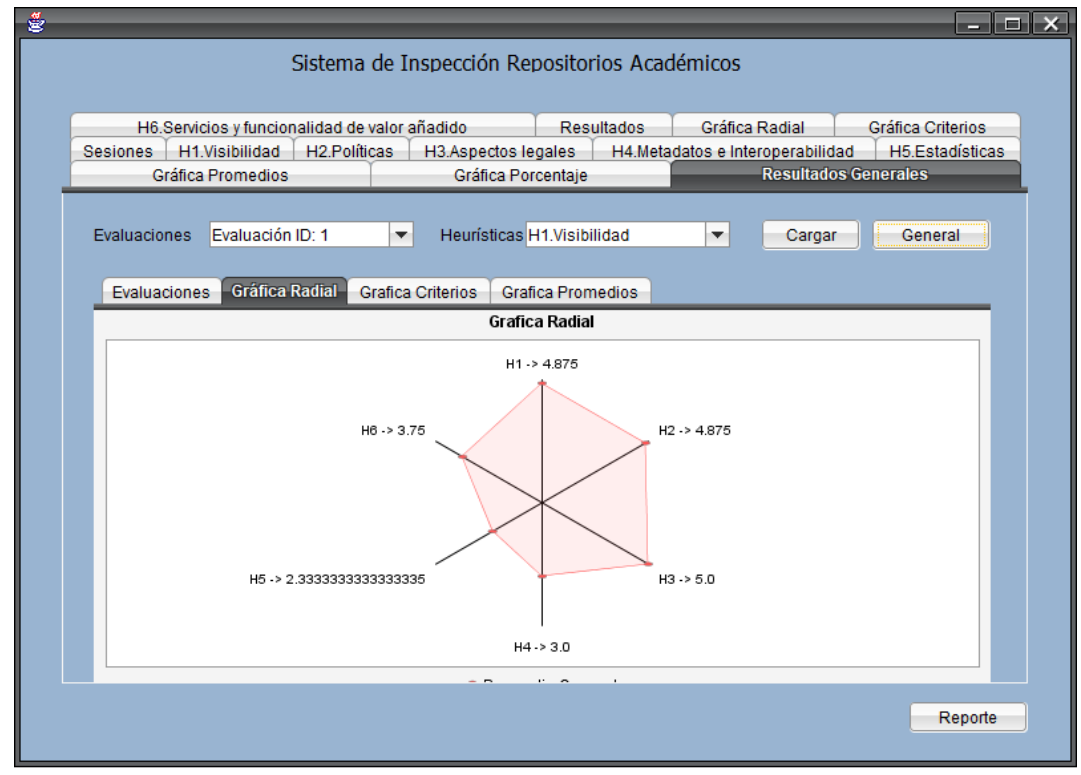

A continuación, en la Tabla 4 se presentan las recomendaciones obtenidas a partir de la prueba de aquellas heurísticas con porcentaje de cumplimiento menor o igual al $75 \%$.

Tabla 4

Recomendaciones obtenidas para el

repositorio de la Universidad de Lima

\begin{tabular}{|l|l|}
\hline \multicolumn{1}{|c|}{ Heurísticas } & \multicolumn{1}{c|}{ Recomendaciones } \\
\hline H4. Metadatos & $\begin{array}{l}\text { Se recomienda la inclusión del campo dc:relation para aquellos contenidos que han contado con } \\
\text { financiaciones del estado (C2). } \\
\text { Se recomienda la integración de más contenidos a partir de otros sistemas de la institución (C3) }\end{array}$ \\
\hline $\begin{array}{l}\text { H5. } \\
\text { Interoperabilidad }\end{array}$ & $\begin{array}{l}\text { Se recomienda incluir la funcionalidad de estadísticas anuales de los diferentes tipos de } \\
\text { contenidos (C2). } \\
\text { Se recomienda ubicar la opción estadística en una región de mayor jerarquía visual (C3). }\end{array}$ \\
\hline H6. Estadísticas & $\begin{array}{l}\text { Se recomienda además de Mendeley agregar otros gestores de referencia tales como RefWork, } \\
\text { Zotero. }\end{array}$ \\
\hline
\end{tabular}

\section{Conclusiones y trabajos futuros}

En este estudio se propusieron un conjunto de heurísticas y criterios de usabilidad aplicables al contexto de los repositorios institucionales. Los criterios generados fueron planteados en términos de preguntas y permiten verificar el cumplimiento de cada una de las heurísticas propuestas. En este sentido, las heurísticas generadas pueden ser empleadas en el proceso de diseño, desarrollo y evaluación de los repositorios, de tal modo se contribuya a la mejora de la interacción con el usuario final.

Las heurísticas y criterios propuestos permiten especificar y precisar la conducción de evaluaciones de usabilidad sobre repositorios académicos con respecto a la propuesta desarrollada en Azorín Millaruel et al. (2017). Del mismo modo, estos criterios y heurísticas permiten complementar las evaluaciones e instrumentos convencionales de usabilidad, los cuales no consideran las particularidades de los repositorios académicos institucionales (Hurtado et al., 2018). 
La herramienta propuesta en este trabajo permite automatizar la conducción de inspecciones heurísticas de usabilidad en repositorios institucionales, de tal forma que posibilita el análisis estadístico y gráfico de los resultados obtenidos. Esta herramienta posibilita también la generación de reportes para el posterior estudio por parte de los evaluadores.

A partir de los resultados obtenidos en el caso de estudio, es posible concluir que el repositorio de la Universidad de Lima cumple adecuadamento los aspectos relacionados con la visibilidad en directorios nacionales e internacionales $(\mathrm{H} 1)$, así como la definición clara de la misión y los objetivos (H2), y finalmente, el manejo de licencias y derechos de autor en los contenidos (H3). Del mismo modo, es necesario mejorar algunos aspectos relacionados con las estadisticas por contenido y año (H5), así como la inclusión de contenidos de diferentes dependencias de la institución (H4) y finalmente la vinculación con otros gestores de referencias (H6).

Como trabajo futuro derivado de la presente investigación se pretende ampliar el estudio realizado a otras instituciones académicas en el ámbito del Perú y Colombia. Así mismo, se pretende dotar a la herramienta propuesta de la posibilidad de generar recomendaciones automáticas a partir de los resultados obtenidos en las evaluaciones realizadas.

\section{Referencias bibliográficas}

Azorín Millaruel, C., Bernal Martínez, I., Civico Martín, R., Cózar Santiago, A., Guzmán Pérez, C., Losada Yáñez, M., Morillo Moreno, J. C., Nonó Rius, B., Padrós Cuxart, R., \& Prats Prat, J. (2017). Guía para la evaluación de repositorios institucionales de investigación. http://openaccess.mpg.de/2365/en

Bailey, C. (2005). The role of reference librarians in institutional repositories. Reference Services Review, 33(3), 259-267. https://doi.org/10.1108/00907320510611294

Chanchí, G. E., Campo, W. Y., \& Sierra, L. M. (2019). Estudio del atributo satisfacción en pruebas de usabilidad, mediante técnicas de análisis de sentimientos - ProQuest. Revista Ibérica de Sistemas e Tecnologias de Informação, E23, 340-352.

https://search.proquest.com/openview/dc9c3ac1b6b131619f5c2c7bfa97c1c5/1.pdf?pqorigsite $=$ gscholar $\& \mathrm{cbl}=1006393$

Chanchí, G. E., Gómez Álvarez, M. C., \& Campo Muñoz, W. Y. (2020). Criterios de usabilidad para el diseño e implementación de videojuegos. Revista Ibérica de Sistemas e Tecnologias de Informação, E26, 461-474.

Chao, G. (2009). Human-computer interaction: Process and principles of human-computer interface design. Proceedings - 2009 International Conference on Computer and Automation Engineering, ICCAE 2009, 230233. https://doi.org/10.1109/ICCAE.2009.23

Duperet Cabrera, E., Pérez Martínez, D., Cedeño Rodríguez, M., Ramírez Mustelier, A., \& Montoya Acosta, L. (2015). Importancia de los repositorios para preservar y recuperar la información. MEDISAN, 19(10), 1283. http://scielo.sld.cu/scielo.php?script=sci_arttext\&pid=S1029-30192015001000014

Enriquez, G., \& Casas, S. (2013). Usabilidad en aplicaciones móviles. Informe Científico Técnico UNPA, 5(2), 2547. https://dialnet.unirioja.es/servlet/articulo?codigo=5123524\&info=resumen\&idioma=SPA

Grau, J. (2007). Pensando en el usuario: la usabilidad. Anuario ThinkEPI, 1(1), 172-177. https://dialnet.unirioja.es/servlet/articulo?codigo $=2555899$

Hernández Pérez, T., Mateos, D. R., \& De La Fuente, G. B. (2007). The role of libraries in open access institutional repositories. Anales de Documentación, 10, 185-204. http://europa.eu/scadplus/glossary/lisbon_strategy_es.htm 
Hurtado, S., Pimentel, J., \& Chanchí, G. E. (2018). Estudio comparativo de métodos de evaluación de usabilidad para sitios web. In E. Serna (Ed.), Desarrollo e Innovación en Ingeniería (pp. 129-136). Instituto Antioqueño de Investigación. http://fundacioniai.org/index.php/libros.html

Martins, V. F., De Paiva Guimaraes, M., \& Correa, A. G. (2013). Usability test for Augmented Reality applications. Proceedings of the 2013 39th Latin American Computing Conference, CLEI 2013. https://doi.org/10.1109/CLEl.2013.6670668

Preece, J. (1994). Human Computer Interaction (ICS). Addison Wesley.

Sanchez, W. (2011). La usabilidad en ingeniería de software: definición y características. Ing-Novación, 2, 7-21. http://www.redicces.org.sv/jspui/bitstream/10972/1937/1/2. La usabilidad en Ingenieria de Softwaredefinicion y caracteristicas.pdf

Solis Tech. (2016). Human-Computer Interaction: The Fundamentals Made Easy! (2nd ed.). CreateSpace Independent Publishing Platform.

https://books.google.com.co/books/about/Human_Computer_Interaction.html?id=ujUtjwEACAAJ\&redir_ esc $=y$

Weichbroth, P. (2020). Usability of mobile applications: A systematic literature study. IEEE Access, 8, 5556355577. https://doi.org/10.1109/ACCESS.2020.2981892

Esta obra está bajo una Licencia Creative Commons Attribución-NoCommercial 4.0 International

\section{(cc) EY-NC}

